\title{
INVARIANTS FOR AUTOMORPHISMS OF CERTAIN ITERATED SKEW POLYNOMIAL RINGS
}

\author{
by DAVID A. JORDAN and IMOGEN E. WELLS*
}

(Received 6th June 1994)

\begin{abstract}
Rings of invariants are identified for some automorphisms $\theta$ of certain iterated skew polynomial rings $R$, including the enveloping algebra of $s l_{2}(k)$, the Weyl algebra $A_{1}$ and their quantizations. We investigate how finite-dimensional simple $R$-modules split over the ring of invariants $R^{\theta}$ and how finite-dimensional simple $R^{\theta}$-modules extend to $R$.
\end{abstract}

1991 Mathematics subject classification: 16S36, 17 B37.

\section{Introduction}

In a sequence of papers $[3,4,5,7]$ the first author has studied a class of iterated skew polynomial rings $R$ in two indeterminates $y$ and $x$ over a finitely generated commutative $k$-algebra $A$, where $k$ is an algebraically closed field. The principal example is the enveloping algebra of the Lie algebra $s l_{2}(k)$ [3]. Other examples include the quantized enveloping algebra of $s l_{2}(k)$ [3], the quantized Weyl algebra in two variables $[5,7]$, the coordinate rings for various quantum groups $[3,5,7]$, and the enveloping algebra of the dispin Lie superalgebra [5, 7].

Given a positive integer $n$ not divisible by char $k$, these algebras all admit an automorphism $\theta$ of order $n$ acting as the identity on $A$ and with $y \mapsto \omega y$ and $x \mapsto \omega^{-1} x$, where $\omega \in k$ is a primitive $n$th root of unity. The purpose of this paper is to study the ring of invariants for such automorphisms for a slightly more general iterated skew polynomial ring $R$ in two variables, including, for example, the ordinary Weyl algebra $A_{1}$ as well as the quantized Weyl algebra. The ring of invariants turns out to be a factor of a ring constructed in the same way from the polynomial ring $A[w]$ as $R$ is from $A$. As a consequence, the results in [7] determining the finite-dimensional simple modules over $R$ may be applied to determine the finite-dimensional simple modules over the ring of invariants $R^{0}$. Indeed it is possible to see how each finite-dimensional simple $R$-module splits over $R^{0}$. We shall see that for a certain class of finite-dimensional simple $R$-module $X$, which often yields all the finite-dimensional simple modules, $X$ is the direct sum of $r$ simple $R^{0}$-modules of dimension $q+1$ and, provided $q>0, n-r$ simple $R^{0}$-modules of dimension $q$, where $\operatorname{dim}_{k} X=q n+r, 0 \leq r<n$. From this it follows, for example, that

* With the support of a University of Sheffield Postgraduate Research Scholarship 
if $R=U\left(s l_{2}(k)\right)$ then $R^{\theta}$ has $n^{2}$ simple modules of each positive dimension. This result for $U\left(s l_{2}(k)\right)$ has been obtained independently by Kraft and Small [8].

\section{Basic details of $R$ and $R^{\theta}$}

2.1 The ring $R$. Let $A$ be a finitely generated commutative algebra over an algebraically closed field $k$, let $\alpha$ be a $k$-automorphism of $A$, let $v \in A$ and let $\rho \in k \backslash\{0\}$. Let $S$ be the skew polynomial ring $A[y ; \alpha]$ and extend $\alpha$ to $S$ by setting $\alpha(y)=\rho^{-1} y$. There is an $\alpha^{-1}$-derivation $\delta$ of $S$ such that $\delta(A)=0$ and $\delta(y)=v$. This is a special case of a construction of skew derivations described in [2, 2.8]. The ring $R=R(A, \alpha, v, \rho)$ is the skew polynomial ring $S\left[x ; \alpha^{-1}, \delta\right]$. Thus $x y-\rho y x=v$ and, for all $a \in A$, $x a=\alpha^{-1}(a) x$ and $y a=\alpha(a) y$.

This notation will be fixed throughout the paper.

2.2 Casimir element. If $v=u-\rho \alpha(u)$ for some $u \in A$ then the element $z=x y-u=$ $\rho(y x-\alpha(u))$ is a normal element of $R$ inducing a $k$-automorphism $\beta$ of $R$ such that $\beta(a)=a$ for all $a \in A, \beta(y)=\rho y$ and $\beta(x)=\rho^{-1} x$. We shall say that the 4-tuple $(A, \alpha, v, \rho)$ is conformal when $v$ has this form $u-\rho \alpha(u)$. Examples of rings which arise in the conformal case are listed in [3], where $\rho=1$, and [7]. If $\rho=1$ then $z$ is central and if $\rho$ is an $n$th root of unity then $z^{n}$ is central. In the general case, we set $w=x y=\rho y x+v$. Then $a w=w a$ for all $a \in A$ and when $v$ is of the form $u-\rho \alpha(u)$, $w=z+u$ and $A[z]=A[w]$.

We extend $\alpha$ to a $k$-automorphism, also denoted $\alpha$, of the ring $A[w]$ by setting $\alpha(w)=\rho^{-1}(w-v)$ and $\alpha^{-1}(w)=\rho w+\alpha^{-1}(v)$. In the conformal case, $\alpha(z)=\rho^{-1} z$ and, in general, $y w=\alpha(w) y$ and $x w=\alpha^{-1}(w) x$.

In the conformal case, the normal element $z$ generates a non-zero proper ideal of $R$ which consequently is not simple. The most obvious example of a ring of the form $R$ in which $v$ is not of the form $u-\rho \alpha(u)$ is the Weyl algebra $A_{1}$ where $A=k, \alpha=\mathrm{id}$, $\rho=1$ and $v=1$ whereas $u-\rho \alpha(u)=0$ for all $u \in A$. The ring $A_{1}$ is well known to be simple if and only if char $k=0$. Conditions for $R$ to be simple will be discussed in [11].

2.3 Identities. Set $v_{0}=0$ and, for $m \geq 1, v_{m}=\sum_{j=0}^{m-1} \rho^{j} \alpha^{j}(v)$. It is easy to see that, for all $m, m^{\prime} \in \mathbb{N}, v_{m}+\rho^{m} \alpha^{m}\left(v_{m^{\prime}}\right)=v_{m+m^{\prime}}$ and that, in the conformal case, $v_{m}=$ $u-\rho^{m} \alpha^{m}(u)$. The following identities, which hold for $m \geq 1$, can be checked inductively. In (3) $-(6), \alpha$ is extended to $A[w]$ as in 2.2 .

(1) $x y^{m}-\rho^{m} y^{m} x=v_{m} y^{m-1}$.

(2) $x^{m} y-\rho^{m} y x^{m}=\alpha^{1-m}\left(v_{m}\right) x^{m-1}$.

(3) $x^{m} y^{m}=\prod_{j=0}^{m-1} \alpha^{-j}(w)$.

(4) $y^{m} x^{m}=\prod_{j=1}^{m} \alpha^{j}(w)$.

(5) $\alpha^{m}(w)=\rho^{-m}\left(w-v_{m}\right)$.

(6) $\alpha^{-m}(w)=\rho^{m} w+\alpha^{-m}\left(v_{m}\right)$.

Note that $(1)$ is equivalent to saying that $\alpha^{-1}\left(y^{m}\right)=\rho^{m} y$ and $\delta\left(y^{m}\right)=v_{m} y^{m-1}$. 
2.4 Grading. Every element of $R$ is a unique $A$-linear combination of the elements $y^{i} x^{j}, i \geq 0, j \geq 0$. For $m \in \mathbb{Z}$, let $R_{m}$ be the set of all $A$-linear combinations of the elements $y^{i} x^{j}$ with $i-j=m$. Because of the relations used to define $R$ as a ring extension of $A, R=\bigoplus_{m \in \mathbf{Z}} R_{m}$ is a $\mathbb{Z}$-graded ring. Note that $w \in R_{0}$. By 2.3 (3) and (4), $R_{0}=A[w]$, while if $m>0$ then $R_{m}=y^{m} A[w]=A[w] y^{m}$ and $R_{-m}=x^{m} A[w]=A[w] x^{m}$. Note that $R_{m}$ can also be described as the set of all $A$-linear combinations of the elements $x^{i} y^{j}$ with $j-i=m$. In the conformal case, $R_{m}=A[z] y^{m}$ or $A[z] x^{-m}$ as appropriate.

2.5 The factor ring $T$. Suppose that $(A, \alpha, v, \rho)$ is conformal. We shall denote the factor ring $R / z R$, which was studied in [4] and [6], by $T$ or, more explicitly, $T(A, \alpha, u)$. It is the ring extension of $A$ generated by $X$ and $Y$, the images of $x$ and $y$ respectively, subject to the relations

$$
X Y=u, \quad Y X=\alpha(u)
$$

and, for all $a \in A$,

$$
Y a=\alpha(a) Y, \quad X a=\alpha^{-1}(a) X .
$$

Note that $T$ is independent of the parameter $\rho$. As the normal element $z$ is homogeneous of degree 0 , there is an induced $\mathbb{Z}$-grading on $T, T=\bigoplus_{m \in \mathbf{Z}} T_{m}$, where $T_{0}=A$, and, for $m>0, T_{m}=Y^{m} A=A Y^{m}$ and $T_{-m}=X^{m} A=A X^{m}$. Each element has a canonical form

$$
a_{n} X^{n}+\ldots+a_{1} X+a_{0}+b_{1} Y+\ldots+b_{m} Y^{m}, \quad \text { where } a_{i}, b_{j} \in A
$$

2.6 The automorphism $\theta$ of $R$ and its invariants. Let $n$ be a positive integer and let $\omega \in k$ be a primitive $n$th root of unity. Note that $n$ must be invertible in $k$. There is a $k$ automorphism $\theta$ of $R$ acting as the identity on $A$ and with $\theta(y)=\omega y$ and $\theta(x)=\omega^{-1} x$. Clearly $\theta$ has order $n$ and, for the grading described in $2.4, \theta$ is a graded automorphism. It is easy to identify the ring $R^{0}$ of invariants in terms of this grading. For each $m, \theta$ acts on $R_{m}$ by multiplication by $\omega^{m}$. Thus

$$
R^{0}=\bigoplus_{j \in \mathbf{Z}} R_{n j}
$$

that is,

$$
R^{0}=\left(\bigoplus_{j \geq 1} A[w] y^{n j}\right) \bigoplus A[w] \bigoplus\left(\bigoplus_{j \geq 1} A[w] x^{n j}\right)
$$

Writing $B$ for the polynomial ring $A[w]$,

$$
R^{0}=\left(\bigoplus_{j \geq 1} B Y^{j}\right) \bigoplus B \bigoplus\left(\bigoplus_{j \geq 1} B X^{j}\right)
$$


By 2.2(3) and (4), $X Y=U_{n}$ and $Y X=\alpha^{n}\left(U_{n}\right)$, where $U_{n}=\prod_{j=0}^{n-1} \alpha^{-j}(w)$ so that $\alpha^{n}\left(U_{n}\right)=\prod_{j=1}^{n} \alpha^{j}(w)$.

It follows that there is a surjective graded homomorphism $\chi: T\left(B, \alpha^{n}, U_{n}\right) \rightarrow R^{0}$ given by $\chi(X)=x^{n}$ and $\chi(Y)=y^{n}$. Given that $\chi$ is graded, it is easily seen to be an isomorphism. Thus we have the following result.

Theorem. The fixed ring $R^{0}$ of $R$ for $\theta$ is isomorphic to $T\left(B, \alpha^{n}, U_{n}\right)$.

In the remainder of the paper we shall use the isomorphism $\chi$ to identity $R^{0}$ with $T\left(B, \alpha^{n}, U_{n}\right)$. This includes the case $n=1$ which gives a different way of viewing the ring $R$.

Corollary. $R(A, \alpha, v, \rho)=T(A[w], \alpha, w)$, where $\alpha$ is extended to $A[w]$ by setting $\alpha(w)=\rho^{-1}(w-v)$.

This corollary gives a way of generalizing known results from the conformal case with $\rho=1$ to the general case. In 3.7 we shall do this for a result from [3] on extensions of simple modules.

2.7 The automorphism $\theta$ of $T$ and its invariants. Consider a $k$-algebra of the form $T=T(A, \alpha, u)=R / z R$ where $R=R(A, \alpha, u-\alpha(u), 1)$. The automorphism $\theta$ of $R$ introduced in 2.6 fixes $z$ and induces a $k$-automorphism of order $n$, also to be denoted $\theta$, of $T$ acting as the identity on $A$ and with $\theta(Y)=\omega Y$ and $\theta(X)=\omega^{-1} X$. It is easy to see that the fixed ring is the ring extension of $A$ generated by $Y^{n}$ and $X^{n}$ subject to the relations

$$
X^{n} Y^{n}=u_{n}, \quad Y^{n} X^{n}=\alpha^{n}\left(u_{n}\right),
$$

where $u_{n}=\prod_{j=0}^{n-1} \alpha^{-j}(u)$. Hence $T^{0}$ can be identified with $T\left(A, \alpha^{n}, u_{n}\right)$, where the indeterminates are written as $Y^{n}$ and $X^{n}$ rather than $Y$ and $X$.

When $R$ is identified with $T(A[w], \alpha, w)$ using Corollary 2.6, the automorphism $\theta$ of $R$ becomes the automorphism $\theta$ of $T(A[w], \alpha, w)$ as above, that is $\theta$ acts as the identity on $A[w], \theta(Y)=\omega Y$ and $\theta(X)=\omega^{-1} X$. The descriptions in Theorem 2.6 and above of the invariants for $R$ and rings of the form $T$ both give the ring of invariants of $R=T(A[w], \alpha, w)$ to be $T\left(A[w], \alpha^{n}, U_{n}\right)$. As it is easier to work with rings of the form $T$ than those of the form $R$, we shall often work with a general ring $T$ and then use Corollary 2.6 to apply results to the general ring $R(A, \alpha, v, \rho)$.

2.8 Examples. One case for which the fixed ring already appears in the literature is when $A=k, \rho=1=v$ and $\alpha=$ id so that $R$ is the Weyl algebra $A_{1}$. In the notation of this paper, the fixed ring is isomorphic to $T\left(k[w], \alpha^{n}, \prod_{j=0}^{n-1}(w+j)\right)$ where $\alpha(w)=w-1$. The algebras similar to the enveloping algebra of $s l_{2}(k)$ which are the subject of [10] are of the form $R=R(A, \alpha, v, \rho)$ with $A=k[w]$ and in [10] the fixed ring of the automorphism $\theta$ of $A_{1}$ is identified as a factor of such an algebra. 
For another example, take $A=k[t], \alpha(t)=t+2, u=-\frac{1}{4}(t-1)^{2}$ and $\rho=1$ so that $v=u-\rho \alpha(u)=t$. In this case, $R$ is the enveloping algebra of the Lie algebra $s l_{2}(k)$. (In the more standard notation for this algebra, $x, y$ and $t$ are written as $e, f$ and $h$.) Here $\alpha$ extends to $A[w]=k[t, w]$ with $\alpha(w)=w-t$ and $\alpha^{-1}(w)=w+t-2$. The element $U_{n}$ in 2.6 is $\prod_{j=0}^{n-1}\left(w+j t-j^{2}-j\right)$ and the fixed ring of $\theta$ is isomorphic to $T\left(k[t, w], \alpha^{n} . U_{n}\right)$.

Finally, suppose that char $k \neq 2$ and take $A=k[t], \alpha(t)=t+1, \rho=-1$ and $u=(2 t-1) / 4$ so that $v=u+\alpha(u)=t$. When $k=\mathbb{C}$, the ring $R$ is the universal enveloping algebra of the dispin Lie superalgebra $B[0,1]$, see [7, Example 1.3]. Here $\alpha$ extends to $A[w]=k[t, w]$ with $\alpha(w)=t-w$ and $\alpha^{-1}(w)=t-w-1$. The fixed ring of $\theta$ is isomorphic to $T\left(k[t, w], \alpha^{n}, U_{n}\right)$, where, in the formula, $U_{n}=\prod_{j=0}^{n-1} \alpha^{-j}(w)$,

$$
\alpha^{-j}(w)= \begin{cases}w-\frac{j}{2} & \text { if } j \text { is even } \\ -w+t-\frac{j+1}{2} & \text { if } j \text { is odd }\end{cases}
$$

\section{Finite-dimensional simple modules}

3.1 Finite-dimensional simple $R$-modules. If $\alpha^{s}(I)=I$ for an ideal $I$ of $A$ or $A[w]$ and some positive integer $s$ then we say that $I$ is periodic and call the least such $s$ the order of $I$.

Theorem. (a) Let $R=R(A, \alpha, v, \rho)$. Every finite-dimensional simple $R$-module is isomorphic to one of the following:

(i) the d-dimensional module $L(M)=R /\left(M R+x R+y^{d} R\right)$ for each maximal ideal $M$ of $A$ containing $v_{d}$ for some (minimal) $d>0$;

(ii) the s-dimensional modules $R /\left(N R+\left(y^{s}-\xi\right) R\right)$ and $R /\left(N R+\left(x^{s}-\xi\right) R\right)$ for each periodic maximal ideal $N$ of $A[w]$ of order $s$ and each $0 \neq \xi \in k$.

(b) Let $T=T(A, \alpha, u)$. Every finite-dimensional simple $R$-module is isomorphic to one of the following:

(i) the d-dimensional module $\mathcal{L}(M)=T /\left(M T+X T+Y^{d} T\right)$ fr each maximal ideal $M$ of $A$ containing $u$ and $\alpha^{d}(u)$ for some minimal $d>0$;

(ii) the s-dimensional modules $T /\left(M T+\left(Y^{s}-\xi\right) T\right)$ and $T /\left(M T+\left(X^{s}-\xi\right) T\right)$ for each periodic maximal ideal $M$ of $A$ of order s and each $0 \neq \xi \in k$.

Proof. (a) is proved in [7] for the conformal case. We shall deduce (b) from the conformal case of (a) and the general case of (a) from (b).

Recall that $T=R / z R$, where $R=R(A, \alpha, u-\alpha(u), 1)$ and $z=x y-u$. The simple $R$-modules are then as given in (a). A simple $R$-module of type (i) is annihilated by $z$ if and only if $u \in M$. Here $v_{d}=u-\alpha^{d}(u)$ and so those simple $R$-modules $L(M)$ which are also simple $T$-modules are those with $u \in M$ and $\alpha^{d}(u) \in M$. The periodic maximal ideals of $A[w]=A[z]$ for which the simple $R$-module of type (ii) gives rise to a simple $T$-module are of the form $M A[z]+z A[z]$ where $M$ is a periodic maximal ideal of $A$. Thus (b) follows from the conformal case of (a).

For the general case of (a), recall from Corollary 2.6 that $R(A, \alpha, v, \rho)=$ 
$T(A[w], \alpha, w)$. For each $d \geq 1$, there is a bijection between the maximal ideals of $A$ containing $v_{d}$ and the maximal ideals of $A[w]$ containing $w$ and $\alpha^{d}(w)$ given by $M \mapsto$ $M A[w]+w A[w]$. Also, if $T^{\prime}=T(A[w], \alpha, w)$ then, because $w=X Y, \quad(M A[w]+$ $w A[w]) T^{\prime}+X T^{\prime}+Y^{d} T^{\prime}=M T^{\prime}+X T^{\prime}+Y^{d} T^{\prime}$. The general case of (a) then follows from (b).

Note that if $A$ has no periodic maximal ideals, as in the cases of the enveloping algebras of the Lie algebra $s l_{2}(k)$ and the dispin Lie superalgebra when char $k=0$, only type (i) occurs.

3.2 Finite-dimensional simple $T^{\theta}$-modules. Let $T=T(A, \alpha, u)$, let $n$ be a positive integer and let $\theta$ be the automorphism of $T$ introduced in 2.7. Thus $T^{0}=T\left(A, \alpha^{n}, u_{n}\right)$, where $u_{n}=\prod_{j=0}^{n-1} \alpha^{-j}(u)$ and the indeterminates are $Y^{n}$ and $X^{n}$ rather than $Y$ and $X$.

Theorem 3.1(b) gives the following classification of the finite-dimensional simple $T^{0}$-modules. Every finite-dimensional simple $T^{0}$-module is isomorphic to one of the following:

(i) the $d$-dimensional module $\mathcal{L}_{0}(N)=T^{0} /\left(N T^{0}+X^{n} T^{0}+Y^{n d} T^{0}\right)$ for each maximal ideal $N$ of $A$ containing $u_{n}$ and $\alpha^{n d}\left(u_{n}\right)$ for some (minimal) $d>0$;

(ii) the $s$-dimensional modules $T^{0} /\left(N T^{0}+\left(Y^{n s}-\xi\right) T^{0}\right)$ and $T^{0} /\left(N T^{0}+\left(X^{n s}-\xi\right) T^{0}\right)$ for each periodic maximal ideal $N$ of $A$ of order $s$ under $\alpha^{n}$ and each $0 \neq \xi \in k$.

A similar classification for the finite-dimensional simple $R^{0}$-modules can be derived using Theorem 2.6.

We next analyse how the finite-dimensional simple $T$-modules split over $T^{0}$.

Theorem. Let $M$ be a maximal ideal of $A$ giving rise to a d-dimensional simple $T$-module $\mathcal{L}(M)=T /\left(M T+X T+Y^{d} T\right)$ as in 3.1(a) (i). Write $d=q n+r$ where $0 \leq r<n$. Then $\mathcal{L}(M)$ is isomorphic to the direct sum of $r$ simple $T^{0}$-modules $\mathcal{L}_{0}\left(\alpha^{-i}(M)\right), 0 \leq i<r$, of dimension $q+1$ and, if $q>0, n-r$ simple $T^{0}$-modules $\mathcal{L}_{0}\left(\alpha^{-i}(M)\right), r \leq i<n$, of dimension $q$. Furthermore every finite-dimensional $T^{0}$-module of the form $\mathcal{L}_{0}(N)$ occurs as a $T^{0}$-summand of a simple $T$-module $\mathcal{L}\left(\alpha^{i}(N)\right)$ for some $i$.

Proof. Recall that $d>0$ is minimal with $u \in M$ and $\alpha^{d}(u) \in M$. For $0 \leq i \leq n-1$, set $N_{i}=\alpha^{-i}(M)$. Thus $u_{n} \in N_{i}$ for each $i$. Note that $\alpha^{n q}\left(u_{n}\right)=\prod_{j=0}^{n-1} \alpha^{n q-j}(u)$ and $\alpha^{n(q+1)}\left(u_{n}\right)=\prod_{j=1}^{n} \alpha^{n q+j}(u)$. Let $0 \leq i<r$. Then $\quad \alpha^{n q+(r-i)}(u)=\alpha^{d-i}(u) \in N_{i} \quad$ and so $\alpha^{n(q+1)}\left(u_{n}\right) \in N_{i}$. Moreover, $q+1$ is the least positive integer $j$ such that $\alpha^{n j}\left(u_{n}\right) \in N_{i}$. Thus there is a $q+1$-dimensional simple $T^{0}$-module $\mathcal{L}_{0}\left(N_{i}\right)$. Now suppose that $r \leq i<n$. Notice that this implies that $q>0$ and $d \geq n$. Then $\alpha^{n q-(i-r)}(u)=\alpha^{d-i}(u) \in N_{i}$ and so $\alpha^{n q}\left(u_{n}\right) \in N_{i}$. Furthermore, $q$ is the least positive integer $j$ such that $\alpha^{n j}\left(u_{n}\right) \in N_{i}$. Hence there is a $q$-dimensional simple $T^{0}$-module $\mathcal{L}_{0}\left(N_{i}\right)$. Thus, unless $q=0$, in addition to the above $r$ simple $T^{0}$-modules of dimension $q+1, M$ gives rise to $n-r$ simple $T^{0}$ modules of dimension $q$.

We next show that, over $T^{0}$, the simple $T$-module $\mathcal{L}(M)$ splits as the direct sum of the simple modules $\mathcal{L}_{0}\left(N_{i}\right), 1 \leq i \leq m$, where $m$ is the minimum of $n$ and $d$. For $0 \leq j \leq d-1$, let $b_{j}=Y^{j}+\left(M T+X T+Y^{d} T\right)$. Then $\left\{b_{j}\right\}_{0 \leq j \leq d-1}$ is a basis for $\mathcal{L}(M)$ and each $A b_{j}=b_{j} A$ is a one-dimensional $A$-submodule of $\mathcal{L}(M)$ with $\operatorname{ann}_{A} b_{j}=\alpha^{-j}(M)$. The 
action of $X$ and $Y$ is given by the rules $b_{0} X=0=b_{d-1} Y, b_{j} Y=b_{j+1}$ for $0 \leq j \leq d-2$ and, because $Y X=\alpha(u), b_{j} X=b_{j-1} \alpha(u)$ for $1 \leq j \leq d-1$.

Fix $0 \leq i<m$ and let $e_{i}=\operatorname{dim} \mathcal{L}_{0}\left(N_{i}\right)$. Thus $e_{i}=q+1$ if $0 \leq i<r$ and $e_{i}=q$ if $q>0$ and $r \leq i<n$. Then $\mathcal{L}_{0}\left(N_{i}\right)$ has a basis $\left\{c_{i j}\right\}_{0 \leq j \leq e_{j}-1}$ analogous to the basis $\left\{b_{j}\right\}$ of $\mathcal{L}(M)$. Thus $c_{i j}=Y^{n j}+\left(M T^{0}+X^{n} T^{0}+Y^{n e_{i}} T^{0}\right)$, each $A c_{i j}=c_{i j} A$ is a one-dimensional $A$-submodule of $\mathcal{L}_{0}\left(N_{i}\right), \quad \operatorname{ann}_{A} c_{i j}=\alpha^{-j n}\left(N_{i}\right), \quad c_{i 0} X^{n}=0=c_{i, d-1} Y^{n}, \quad c_{i j} Y^{n}=c_{i, j+1} \quad$ for $0 \leq j \leq e_{i}-2$, and $c_{i j} X^{n}=b_{j-1} \alpha^{n}\left(u_{n}\right)$ for $1 \leq j \leq e_{i}-1$. Now let $L_{i}$ be the subspace $\bigoplus_{j=0}^{e_{i}-1} b_{i+j n} A=b_{i} A+b_{i+n} A+\ldots b_{i+\left(e_{i}-1\right) n} A$ of $\mathcal{L}(M)$. As $T^{0}$ is generated, as a ring extension of $A$, by $Y^{n}$ and $X^{n}$, each $L_{i}$ is an $e_{i}$-dimensional $T^{0}$-submodule of $L(M)$. Clearly $L(M)=\bigoplus_{i=0}^{m-1} L_{i}$.

Each $L_{i}$ has basis $\left\{b_{i+j n}\right\}_{0 \leq j \leq e_{i}-1}$ and ann $b_{i+j n}=\alpha^{-(i+j n)}(M)=\alpha^{-n j}\left(N_{i}\right)=\operatorname{ann}_{A} c_{i j}$. Also $b_{i} X^{n}=0=b_{i+\left(e_{i}-1\right) n} Y^{n}$ and $b_{j+j n} Y^{n}=b_{i+(j+1) n}$ for $0 \leq j \leq e_{i}-2$. If $1 \leq j \leq e_{i}-1$ then $b_{i+j n} X^{n}=b_{i+(j-1) n} \alpha(u) \alpha^{2}(u) \ldots \alpha^{n}(u)=b_{i+(j-1) n} \alpha^{n}\left(u_{n}\right)$. Hence there is a $T^{0}$-isomorphism $f: L_{i} \rightarrow \mathcal{L}_{\theta}\left(N_{i}\right)$ given by $f: b_{i+j n} \mapsto c_{i j}$. Identifying each $L_{i}$ with $f\left(L_{i}\right), \mathcal{L}(M)$ splits over $T^{0}$ as claimed.

Now let $N$ be a maximal ideal of $A$ for which there is a finite-dimensional simple $T^{0}$-module $\mathcal{L}_{0}(N)$. Then $u_{n}=\prod_{j=0}^{n-1} \alpha^{-j}(u) \in N$ and $\operatorname{dim} \mathcal{L}_{0}(N)$ is the minimal positive integer $e$ such that $\alpha^{n e}\left(u_{n}\right) \in N$. As $u_{n} \in N$ there exists a minimal integer $j_{1}$ such that $0 \leq j_{1} \leq n-1$ and $u \in \alpha^{j_{1}}(N)$. Let $M=\alpha^{j_{1}}(N)$. Also there exists a maximal integer $j_{2}$ with $0 \leq j_{2} \leq n-1$ and $u \in \alpha^{j_{2}-n e}(N)$. If $d=n e-j_{2}+j_{1}$ then $\alpha^{d}(u) \in \alpha^{j_{1}}(N)$ and, by the choice of $e, j_{1}$ and $j_{2}, d$ is the least positive integer with $\alpha^{d}(u) \in M$. Thus there is a $d$ dimensional simple $T$-module $\mathcal{L}(M)$ and, in the above notation, $N=N_{j_{1}}$ is a $T^{0}$ summand of $\mathcal{L}(M)$.

3.3 Finite-dimensional simple $R$-modules and $\boldsymbol{R}^{\theta}$-modules. Let $\theta$ be the automorphism of $R$ introduced in 2.6. Thus $R^{0}=T\left(A[w], \alpha^{n}, U_{n}\right)$. Theorem 3.2 can be applied to the finite-dimensional simple $R$-modules and $R^{0}$-modules.

Corollary. Let $M$ be a maximal ideal of $A$ giving rise to a d-dimensional simple $R$ module $L(M)=R /\left(M R+x R+y^{d} R\right)$ as in 3.1(a) (i). Write $d=q n+r$, where $0 \leq r<n$. Then $L(M)$ is isomorphic to the direct sum of $r$ simple $R^{\theta}$-modules $\mathcal{L}\left(\alpha^{-i}(\bar{M} A[w]+\right.$ $w A[w])), \quad 0 \leq i<r$, of dimension $q+1$ and, if $q>0, n-r$ simple $R^{0}$-modules $\mathcal{L}\left(\alpha^{-i}(M A[w]+w A[w])\right), r \leq i<n$, of dimension $q$. Furthermore every finite-dimensional $R^{0}$-module of the form $\mathcal{L}(N)$ occurs as an $R^{0}$-summand of a simple $R$-module $L\left(\alpha^{i}(N) \cap A\right)$ for some $i$.

Proof. When $R$ is identified with $T(A[w], \alpha, w)$ as in $2.6, L(M)$ becomes $\mathcal{L}(M A[w]+w A[w])$, see 3.1 . The result follows on applying Theorem 3.2 to $T(A[w], \alpha, w)$.

3.4 Examples. With $n$ and $\theta$ as in 2.6, we discuss three examples in which $A$ has no periodic maximal ideals so that, by 3.1 and 3.3 , every finite-dimensional $R$-module has the form $L(M)$ and splits as the direct sum of $r$ simple $R^{0}$-modules of dimension $q+1$ and, if $q>0, n-r$ simple modules of dimension $q$, where $\operatorname{dim} L(M)=q n+r$ and $0 \leq r<n$. All finite-dimensional simple $R^{0}$-modules occur in this way. 
(i) As is well-known, or can be seen from the classification in 3.1 (see $[3,3.17]$ for the details), if char $k=0$ then $R=U\left(s l_{2}(k)\right)$ has a unique $d$-dimensional simple module $L_{d}$ for each positive integer $d$. Fix a positive integer $j$. The values of $d$ for which $L_{d}$ has $j$-dimensional simple $R^{0}$-summands are $d=n(j-1)+s, 1 \leq s \leq n$, in which case there are $s$ such summands, and $d=n j+s, 1 \leq s \leq n$, in which case there are $n-s$. Thus the number of $j$-dimensional simple modules is $\sum_{s=1}^{n}(s+n-s)=n^{2}$. This result has been obtained independently by $\mathrm{Kraft}$ and Small, [8, Example 3].

(ii) The quantized enveloping algebra $U_{q}\left(s l_{2}(k)\right)$ is a ring of the form $R$ and, provided $q$ is not a root of unity, has four $d$-dimensional simple modules for each positive integer $d$. See $[3,3.19]$ for details. A similar calculation to the above shows that $R^{0}$ has $4 n^{2}$ simple modules of dimension $j$ for each positive integer $j$.

(iii) Here we consider the case where $R$ is the enveloping algebra of the dispin Lie superalgebra and char $k=0$. Thus $A=k[t], \alpha(t)=t+1$ and $\rho=-1$. Then $R$ has a unique $d$-dimensional simple module $L_{d}$ for each odd positive integer $d$ and no simple modules of even degree. See $[7,4.2]$ for details.

Suppose that $n=2 m-1$ is odd. Fix an odd positive integer $j$. The values of $d$ for which $L_{d}$ has $j$-dimensional simple $R^{0}$-summands are $d=n(j-1)+s, 1 \leq s \leq n, s$ odd, in which case there are $s$ such summands, and $d=n j+s, 1 \leq s \leq n$, $s$ even, in which case there are $n-s$. This gives a total of $2 m^{2}-2 m+1$ simple modules of each odd dimension $j$. A similar calculation shows that there are $2 m^{2}-2 m$ simple modules of each even dimension $j$.

Now suppose that $n=2 m$ is even and fix a positive integer $j$. The values of $d$ for which $L_{d}$ has $j$-dimensional simple $R^{\theta}$-summands are $d=n(j-1)+s, 1 \leq s \leq n, s$ odd, in which case there are $s$ such commands, and $d=n j+s, 1 \leq s \leq n, s$ odd, in which case there are $n-s$. This gives a total of $2 m^{2}$ simple modules of each dimension $j$, whether $j$ is odd or even.

3.5 Extending simple $T^{\theta}$-modules to $T$. Let $N$ be a maximal ideal of $A$ for which there is a finite-dimensional simple $T^{0}$-module $\mathcal{L}_{0}(N)$. As shown in 3.2 , there exists a maximal ideal $M$ of $A$ such that $\mathcal{L}_{0}(N)$ is a direct summand, over $T^{0}$, of the simple $T$-module $L(M)$. It is reasonable to ask whether $\mathcal{L}_{0}(N) \otimes_{T^{0}} T$ must be isomorphic to $\mathcal{L}(M)$. If, with $e, j_{1}$ and $j_{2}$ as in the proof of Theorem $3.2, j_{1}$ is the unique integer such that $0 \leq j_{1} \leq n-1$ and $u \in \alpha^{j_{1}}(N)$ and $j_{2}$ is the unique integer with $0 \leq j_{2} \leq n-1$ and $u \in \alpha^{j_{2}-n e}(N)$ then the answer is positive. The following example shows that it is not positive in general.

Let char $k=0$, let $A$ be the polynomial ring $k[t, w]$, and let $\alpha$ be the $k$-automorphism of $A$ such that $\alpha(t)=t+1$ and $\alpha(w)=w+t(t-1)(4 t+1)$. Let $n=2$ and let $M$ be the maximal ideal $t A+w A$. Form the ring $T=T(A, \alpha, w)$. Then $w \in M, \alpha(w) \in M$ and $a^{2}(w) \in M \quad$ but $\alpha^{-1}(w)=w-(t-1)(t-2)(-4 t+3) \equiv-6 \bmod M . \quad$ As $w \in M \quad$ and $\alpha(w) \in M$, there is a one-dimensional $T$-module $\mathcal{L}(M)=T /(X T+Y T+M T)$. As a $T^{0}$ module, this is $\mathcal{L}_{0}(M)$, its annihilator in $T^{0}$ is the ideal $X^{2} T^{0}+Y^{2} T^{0}+M T^{0}$ and $\mathcal{L}_{\theta}(M) \otimes_{T^{0}} T \simeq T /\left(X^{2} T+Y^{2} T+M T\right)$. Let $J=X^{2} T+Y^{2} T+M T$. Then $X^{2} Y=X w=$ $\alpha^{-1}(w) X \equiv-6 X \bmod M T$. Hence $X \in J$ and so $J=X T+Y^{2} T+M T$. Also $Y^{2} X=$ $Y \alpha(w)=\alpha^{2}(w) Y \in M T$ and therefore $J=X T+Y^{2} S+M T$, where $S=A[Y ; \alpha]$. From 
this it follows easily that $Y \notin J$. Hence $\mathcal{L}_{0}(M) \otimes_{T^{0}} T \simeq T / J$ is not annihilated by $Y$, is two-dimensional with basis $\{1, \bar{Y}\}$ and is not isomorphic to $\mathcal{L}(M)$.

Note that, by Corollary 2.6, $T=T(k[t, w], \alpha, w)=R(k[t], \alpha, u-\alpha(u), 1)$ where $u=t^{2}(t-1)(t-2)$ so this example answers the corresponding question for rings of the form $R$. The simpler example with $u=t(t-1)(t-2)$ works equally here but the specified example has another role later. If the above example is amended so that $\alpha^{2}(u) \notin M$, for example, by taking $u=t^{2}(t-1)$ and so $\alpha(w)=w+t(3 t+1)$, then $Y \in J$ and $\mathcal{L}_{0} \otimes_{T^{o}} T \simeq \mathcal{L}(M)$. Calculations of this sort are used to establish that $\mathcal{L}_{0}(N) \otimes_{T^{0}} T \simeq \mathcal{L}(M)$ in the case claimed above.

3.6 Other finite-dimensional simple $T$-modules and $\boldsymbol{T}^{\theta}$-modules. If there are periodic maximal ideals in $A$ then there are finite-dimensional simple $T$-modules $S$ not of the form $L(M)$. The way in which these modules split over $T^{0}$ is different to that for those of the form $\mathcal{L}(M)$. In particular, the summands all have the same dimension. Such a module $S$ has one of the forms $T /\left(M T+\left(Y^{s}-\xi\right) T\right)$ or $T /\left(M T+\left(X^{s}-\xi\right) T\right)$ for some periodic maximal ideal $M$ of $A$ of order $s$ and some $0 \neq \xi \in k$. Let $m$ be the highest common factor of $n$ and $s$ and note that $N$ has order $s / m$ under $\alpha^{n}$. Then it can be checked that, as a $T^{0}$-module, $S$ is a direct sum of $m$ simple $T^{0}$-modules, each of dimension $s / m$ and of the form given in 3.2(ii). Also, for each of these $T^{0}$-modules $S^{\prime}$, $S^{\prime} \otimes_{R^{0}} R \simeq S$.

3.7 Semisimplicity of finite-dimensional $R$-modules. Suppose that $A$ has no periodic maximal ideals and let $R=R(A, \alpha, v, \rho)$. In [3, Section 5] it is shown that, in the conformal case with $\rho=1$, all finite-dimensional $R$-modules are semisimple if and only if, for all maximal ideals $M$ of $A$ and all positive integers $d<e$,

$$
u-\alpha^{d}(u) \in M \Rightarrow\left(u-\alpha^{e}(u) \notin M \quad \text { and } \quad M^{2}+\left(u-\alpha^{d}(u)\right) A=M\right) .
$$

It follows from this result and the action of the Casimir element $z$ on the non-split extensions which can occur, that, for $T=T(A, \alpha, u)=R / z R$, all finite-dimensional $T$-modules are semisimple if and only if for all maximal ideals $M$ of $A$ and all positive integers $d<e$,

$$
\left(u \in M \quad \text { and } \quad \alpha^{d}(u) \in M\right) \Rightarrow\left(\alpha^{e}(u) \notin M \quad \text { and } \quad M^{2}+u A+\alpha^{d}(u) A=M\right) .
$$

Applying this to $R=T(A[w], \alpha, w)$, we obtain the following generalization of $[3,5.6]$.

Theorem. Suppose that $A$ has no periodic maximal ideals. All finite-dimensional $R$-modules are semisimple if and only if, for all maximal ideals $M$ of $A$ and positive integers $d<e$,

$$
v_{d} \in M \Rightarrow\left(v_{e} \notin M \text { and } M^{2}+v_{d} A=M\right) \text {. }
$$


Proof. By the above, all finite-dimensional $R$-modules are semisimple if and only if, for all maximal ideals $N$ of $A[w]$ and positive integers $d<e$,

$$
\left(w \in N \quad \text { and } \quad \alpha^{d}(w) \in N\right) \Rightarrow\left(\alpha^{e}(w) \notin N \quad \text { and } \quad N^{2}+w A[w]+\alpha^{d}(w) A[w]=N\right) .
$$

There is a bijection between the set of maximal ideals $N$ of $A[w]$ containing $w$ and the set of maximal ideals $M$ of $A$ given by $N=M A[w]+w A[w] \leftrightarrow M=N \cap A$. As $\alpha^{d}(w)=\rho^{-d}\left(w-v_{d}\right)$ by $2.3(5), \quad$ it is clear that $v_{d} \in M \Leftrightarrow \alpha^{d}(w) \in N$. Also $N^{2}+w A[w]+\alpha^{d}(w) A[w]=N \Leftrightarrow N^{2}+w A[w]+v_{d} A[w]=N \Leftrightarrow M^{2}+v_{d} A=M$. The result follows.

3.8 Example. Suppose that $A$ is the Laurent polynomial ring $k\left[t, t^{-1}\right]$ with $\alpha(t)=q^{2} t$ where $0 \neq q \in k$ is not a root of unity. Thus $A$ is $\alpha$-simple and is a principal ideal domain. Let $v=a t+b$ for some $a, b \in k$ with $a \neq 0$ and consider the ring $R=R(A, \alpha . v, \rho)$ where $\rho=q^{-1}$. For $d \geq 1, v_{d}=\left(1+q+\ldots q^{d-1}\right) a t+\left(1+q^{-1}+\ldots q^{-(d-1)}\right) b$ which generates the maximal ideal $M_{d}=\left(t+q^{d-1} \frac{b}{a}\right) A$. As these maximal ideals are distinct, Theorem 3.7 applies to show that all finite-dimensional $R$-modules are semisimple. A particular case of interest is [7, Example 1.4(ii)] where $q=v^{2}$ and $v=v^{-1}\left(t+\frac{v^{2}}{v^{2}-1}\right)$. This algebra $R$ is the localization at the powers of $t$ of the algebra, first considered by Woronowicz [12], obtained as above but with $A=k[t]$ rather than $k\left[t, t^{-1}\right]$. Alternative proofs of the semisimplicity of the finite-dimensional modules for the localization are given in [12] and [1].

3.9 Semisimplicity of finite-dimensional $\boldsymbol{R}^{\theta}$-modules. Applying the method of 3.7 to the fixed ring $R^{0}=T\left(A[w], \alpha^{n}, U_{n}\right)$ gives that all finite-dimensional $R^{\theta}$-modules are semisimple if and only if, for all maximal ideals $N$ of $A[w]$ and positive integers $d<e$,

$$
\left(U_{n} \in N \quad \text { and } \quad \alpha^{n d}\left(U_{n}\right) \in N\right) \Rightarrow\left(\alpha^{n e}\left(U_{n}\right) \notin N \quad \text { and } \quad N^{2}+U_{n} A[w]+\alpha^{n d}\left(U_{n}\right) A[w]=N\right) .
$$

It can be checked that this criterion is equivalent to the corresponding criterion for the case $n=1$ in the proof of 3.7. Thus all finite-dimensional $R$-modules are semisimple if and only if the same is true for $R^{0}$. The "only if" part of this is true in general for the ring of invariants $S=R^{G}$ of a finite group $G$ of automorphisms of a right Noetherian algebra $R$ provided $|G|$ is invertible in $R$. One proof involves using the trace map, see [9, p. 242], to show that for each right ideal $I$ of $S, I R \cap S=I$. From this it follows that any finite-dimensional $S$-module $S / I$ embeds in the $R$-module $R / I R$. As $R$ is finitely generated as an $R^{0}$-module by $[9,26.13(\mathrm{ii})], R / I R$ is finitedimensional and hence semisimple as an $R^{0}$-module. By $[9,26.13$ (iv)], $R / I R$ is semisimple as an $S$-module and therefore $S / I$ is semisimple. Alternatively, see [8, proof of Proposition 1]. The criterion in 3.7 can fail on either of two counts, $v_{e} \in M$ or $M^{2}+v_{d} A \neq M$. The two give rise to different types of non-split extensions. The first gives rise to non-split extensions of $L(M)$ by $L(N)$ and of $L(N)$ by $L(M)$, where $N=\alpha^{-d}(M)$, and the second gives $\operatorname{Ext}_{R}^{1}(L(M), L(M))$ to be non-zero. See [3, Section 5] 
for details. Although there is a similar dichotomy for $R^{0}$-modules, it is possible, as the next example shows, for $R$ to have the property that $\operatorname{Ext}_{R}^{\prime}(X, X)=0$ for all finitedimensional simple $R$-modules $X$ but for $R^{0}$ to fail to inherit this property.

3.10 Example. Consider the example of 3.5 , that is $R=R(k[t], \alpha, u-\alpha(u), 1)$ where $\alpha(t)=t+1$ and $u=t^{2}(t-1)(t-2)$ or, equivalently, $T=T(k[t, w], \alpha, w)$ with $\alpha(t)=t+1$ and $\alpha(w)=w+t(t-1)(4 t+1)$. As $k[t]$ is $\alpha$-simple it follows that each finitedimensional simple $R$-module has the form $L(M)$ for some maximal ideal $M$ of $A$ containing $v_{d}$ for some positive integer $d$. The two-dimensional module $R / J(=T / J)$ in 3.5 is not semisimple.

Suppose that $\operatorname{Ext}_{R}^{1}(L(M), L(M)) \neq 0$ for some maximal ideal $M$ of $A$. Then for some positive integer $d, v_{d} \in M$ but $M^{2}+v_{d} A \neq M$. As $M / M^{2}$ is one-dimensional, it follows that $v_{d} \in M^{2}$. But $v_{d}-u-\alpha^{d}(u)=4 t^{3}+(6 d-9) t^{2}+\left(4 d^{2}-9 d+4\right) t+\left(d^{3}-3 d^{2}+2 d\right)$ so this cubic and its derivative share a common root which must be

$$
\frac{4 d^{3}-6 d^{2}-11 d+12}{2\left(11-4 d^{2}\right)}
$$

From this it follows that $d$ is a root of the polynomial

$$
64 d^{6}-528 d^{4}+1452 d^{2}-1088=\left(4 d^{2}-11\right)^{3}+243 .
$$

This polynomial has no integer roots and so $\operatorname{Ext}_{R}^{1}(L(M), L(M))=0$.

On the other hand, consider the fixed ring $R^{0}$ in the case $n=2$. Let $N$ be the maximal ideal $w A[w]+t A[w]$ of $A[w]$. Then $U_{2}=w \alpha^{-1}(w) \in N$ and $\alpha^{2}\left(U_{2}\right)=\left(w-v_{2}\right)\left(w-v_{1}\right)=$ $\left(w-u+\alpha^{2}(u)\right)(w-u+\alpha(u)) \in N^{2}$ and so $N^{2}+U_{2} A[w]+\alpha^{2}\left(U_{2}\right) A[w] \subseteq N^{2}+w A[w] \subset N$. It follows that there is a one-dimensional simple $R^{0}$-module $\mathcal{L}(N)$ with $\operatorname{Ext}_{R^{0}}^{\mathrm{l}}(\mathcal{L}(N)$, $\mathcal{L}(N)) \neq 0$.

\section{REFERENCES}

1. A. D. BeLL and S. P. SMITH, Some 3-dimensional skew polynomial rings, preprint, University of Wisconsin and University of Washington.

2. K. R. Goodearl and E. S. Letzter, Prime ideals in skew and q-skew polynomial rings, Mem. Amer. Math. Soc. 109 (1994), no. 521.

3. D. A. JoRDAN, Iterated skew polynomial rings and quantum groups, J. Algebra 156 (1993), 194-218.

4. D. A. Jordan, Krull and global dimension of certain iterated skew polynomial rings. In Abelian Groups and Noncommutative Rings (a collection of papers in memory of Robert B. Warfield, Jr.), Contemp. Math. 130 (1992), 201-213.

5. D. A. Jordan, Height one prime ideals of certain iterated skew polynomial rings, Math. Proc. Cambridge Philos. Soc. 114 (1993), 407-425.

6. D. A. Jordan, Primitivity in skew Laurent polynomial rings and related rings, Math. $Z$. 213 (1993), 353-371. 
7. D. A. Jordan, Finite-dimensional simple modules over certain iterated skew polynomial rings, J. Pure Appl. Algebra 98 (1995), 45-55.

8. H. Kraft and L. Small, Invariant algebras and completely reducible representations, Mathematical Research Letters 1 (1994), 297-307.

9. D. S. Passman, Infinite crossed products (Academic Press, San Diego, London, 1989).

10. S. P. SMith, A class of algebras similar to the enveloping algebra of sl(2), Trans. Amer. Math. Soc. 322 (1990), 285-314.

11. I. E. WeLls, Simplicity in some iterated skew polynomial rings, in preparation.

12. S. L. Woronowicz, Twisted SU(2)-group. An example of a non-commutative differential calculus, Publ. R.M.I.S., Kyoto Univ. 23 (1987), 117-181.

School of Mathematics and Statistics

UNIVERSITY OF SHEFFIELD

THE Hicks BuIldiNg

SHEFFIELD S3 7RH, UK 